\title{
Acceptance of Future Persuasive In-Car Interfaces Towards a More Economic Driving Behaviour
}

\author{
Alexander Meschtscherjakov, David Wilfinger, Thomas Scherndl, Manfred Tscheligi \\ ICT\&S Center, University of Salzburg \\ Sigmund Haffner Gasse 18, 5020 Salzburg, Austria \\ \{alexander.meschtscherjakov, david.wilfinger, thomas.scherndl, manfred.tscheligi\}@sbg.ac.at
}

\begin{abstract}
Measuring user acceptance to avoid system rejection by the users in pre-prototype stage of product development is of high interest for both researchers and practitioners. This is especially true when technology uses strategies of persuasion in an emotional laden environment like the car. This paper presents the results of an online survey aiming at evaluating the acceptance of future persuasive in-car interaction approaches for a more economic driving behaviour. Five different persuasive interface concepts are presented and studied towards their acceptance. The results show an overall acceptance of the system concepts and the usefulness of the presented method. We show that individual expectations of the systems' disturbance and risk have an effect on the acceptance of technology and the behavioural intention to use.
\end{abstract}

\section{Categories and Subject Descriptors}

H.5.2 [Information Interfaces and Presentation]: User Interfaces - evaluation/methodology

\section{General Terms}

Design, Human Factors.

\section{Keywords}

In-car interfaces, economy, technology acceptance, persuasion.

\section{INTRODUCTION}

Similar to every aspect of our lives, the pervasion of the automotive context with advanced technologies is increasing. Having in mind that every year a diversity of technologies is developed and reaches customers and potential users, it is highly interesting for both researchers and practitioners, whether these technologies will be accepted by the target group or not. A lack of acceptance will lead to a rejection of the system by new users and to a strong dislike by existing users of comparable technology, who have a high intention on using newer developments. The risk of rejecting systems, which aim at changing attitudes or behaviour, is even higher in emotion-laden contexts like the car, because driving assistance is easily mistaken as a critique on

Copyright held by author(s)

AutomotiveUI'09, September 21-22, 2009, Essen, Germany

ACM 978-1-60558-571-0/09/009 driving behaviour. Therefore it is of high importance to find issues in system design that decrease the acceptance of novel systems in a very early stage of development.

Energy efficiency in the automotive context is currently in the focus of research and industry activities. Besides technological innovations, the drivers' behaviour is a potential area of improvement. This can be fostered by novel interface concepts, which require the users to accept the new technology in order to make it successful. This paper describes how a scenario-based online questionnaire can be used to evaluate user acceptance of persuasive in-car interfaces in a pre-prototype phase of development.

\section{RELATED WORK}

In the next sections an overview on academic as well as industrial research for novel in-car interfaces, persuasive technology and technology acceptance is given.

\subsection{In-car interfaces}

Research on in-car interfaces has recently gained higher attention in the area of human-computer interaction (HCI) [17]. Novel technologies creating attractive in-car user interfaces have become a great challenge [21]. Ablassmeier et al. [1] state that the growing amount of information in cars makes the development of new strategies to cope with this amount of information for drivers necessary. This is when new interaction technologies (e.g. speech interfaces, olfactory interfaces) can provide new possibilities to handle the complexity of the system. For that purpose, several researchers focus on the integration of multimodal interaction in the car. Siewiorek et al. [22], for example, introduce a companion contextual car driver interface that assists the driver.

Research focusing on in-car interface solutions includes different ways of input and output systems that enable new concepts of driver assistance (for example, recent presented concepts were search based interfaces [9], handwritten input [15] and augmented windshield displays [16]). Since the automotive context is already penetrated by a multitude of interaction systems, one way of assisting the driver is through the augmentation of existing in-car interfaces as suggested by Varhelyi et al. [26]. Their active acceleration pedal increases its resistance when the driver exceeds the speed limit. Another interesting approach is the dynamic speedometer, which integrates current speed limits seamlessly into the dashboard [18].

Research activities on supporting the early stages of in-car ICT development is for instance the CARS (Configurable Automotive Research Simulator) project [14]. It enables the application of incar system prototypes in a driving simulator. The presented work 
uses a different approach and addresses the study of in-car interfaces in a pre-prototype and conceptual phase.

\subsection{Persuasion}

The topic of persuasion has been applied to several areas in the realm of HCI. Fogg [7] coined the term Captology ("computers as persuasive technologies") to describe the area where computing technology (web sites, mobile phones, smart environment, virtual reality, etc.) and persuasion (behaviour change, attitude change, motivation, change in worldview, compliance) overlap. Especially Persuasive Technologies, which are defined by Fogg as any interactive computing system designed to change people's attitudes or behaviours, seems to be a promising approach to be researched in automotive environments, when it comes to fostering a more fuel-efficient driving behaviour.

Lately, there have been several approaches from car manufacturers to include persuasive technologies into cars to facilitate a voluntary change of behaviour, attitude, or both to reduce greenhouse gas emissions. A good overview on recent work on approaches to help people become more fuel-efficient drivers can be found at [10]. Persuasive in-car technologies in the academic realm have been researched by Tester et al. [23] and Pace et al. [20], among others.

\subsection{Technology Acceptance}

User acceptance (UA) can be defined as the demonstrable willingness within a user group to employ information technology for the tasks it is designed to support [5]. UA is vital for the success of any information technology system. This leads to the need of informing ICT development as early as possible with data about the UA of the system under development.

There are a high number of research efforts concerning UA [5]. The widest used model for describing UA is the Technology Acceptance Model (TAM) introduced by Davis [4]. It is based on the prediction of user acceptance prior to a real system usage and was further developed in order to enable the study of technology acceptance in a pre-prototype phase of system development [3]. Venkatesh et al. [27] present another approach of modelling UA by introducing the UTAUT model using eight scales for assessing UA such as performance expectancy and social influence.

An early assessment of UA in the development of ICTs can give advantages on both cost and effort [3]. Davis and Venkatesh propose to use the TAM questionnaire as early in the product development as possible. Based on the original TAM they use three scales to assess UA of systems in a pre-prototype state: Perceived Usefulness (U), Perceived Ease of Use (EOU) and Behavioural Intention of Use (BI). Perceived usefulness (U) describes the extent to which the individual believes that using a system will enhance his/her job performance. Perceived ease of use (EOU) is the extent to which an individual believes using a system will be free of effort. In the original TAM Intention of Use (BI) is a function of U and EOU. In the pre-prototype TAM model $\mathrm{BI}$ is covered by additional items. Davis and Venkatesh argue this by the change of the direct influence of EOU on BI, which becomes non-significant after users gained hands on experience which would have lead to U being the only factor to compute BI with. They showed that the TAM is a reliable and valid tool to predict actual usage behaviour.

Technology acceptance in the vehicle was already addressed by Comte et al. [2] studying driver acceptance of automatic speed limiters. Main goal of their work was to evaluate acceptability and if drivers perceive speed limiters to be effective in reducing accidents. Acceptance of advanced traveller information systems (ATIS) was researched by Wochinger and Boehm-Davis [28] letting users rate qualities of the system based on their own needs. Kantowitz et al. [13] conducted acceptance research in order to inform developers how ATIS had to be designed to fulfil their purpose without causing bad experiences that might keep people from using them. All those approaches show the need for an easy and fast assessment of UA towards technology in the car.

Acknowledging the fact that the spread of ICT with persuasive elements in vehicles will increase in the future, the development of those systems will have to take UA into account as the systems might interfere with the drivers wish not to be controlled [2]. While this applies to all kinds of new technologies, it is of special importance in the car environment, which is safety critical and traditionally laden with emotions. A rejection of a system might have a wide range of effects from an image loss of the brand to a severe loss of security when frustrated drivers drive less safe. Applying strategies of persuasion in the car is highly critical as the car is often a very emotional object for the owner. This can increase the effect of persuasion strategies on user experience factors (e.g. fun/enjoyment, comfort, trust) in both positive and negative ways and influence drivers performance [12]. Therefore systems that aim at in-car persuasion have to be designed with an evaluation of design decisions as early as possible to reduce the risk of negative effects.

\section{RESEARCH GOALS}

Given the above described state-of-the-art, appropriate methods to research future persuasive in-car interfaces in a pre-prototype stage are missing. We intend to fold this gap by our approach. We therefore present the following research goals:

\section{Research Goal 1 (RG1)}

The first research goal was to evaluate the user acceptance of persuasive in-car interfaces that are designed to support a fuelefficient driving style. Based on iterative design in the user centred design cycle, it is valuable for the design of interactive systems to study users' reaction and interaction as early as possible in the development phase. That ideally happens also in the moment of concept creation when a minimal effort was invested in implementing the concepts into prototypes. The presented interface concepts are all in this state.

\section{Research Goal 2 (RG2)}

Based on the measured acceptance of the presented interface concepts the aim of the second research goal was to analyse if other factors have influence on the user acceptance. Specifically, it was researched if there are identifiable influences of driver properties and expectations toward the technology concerning safety, disturbance and assistance. Furthermore, we investigated the effect of driver's general attitude toward technology in cars and sociodemographic variables (age, gender, frequency of driving a car) on the acceptance rating. This kind of information could give additional insights for the development process and the design of system properties for special target groups.

\section{Research Goal 3 (RG3)}

Finally, the third research goal of the presented work was to find out if the usage of an online TAM questionnaire for persuasive incar interfaces in a pre-prototype level would lead to results that support future design decisions. 


\section{METHODOLOGY}

Based on the results Davis and Venkatesh [3] it was decided to use this questionnaire for the assessment of persuasive user interfaces for the vehicle in a pre-prototype state. Five interfaces that were designed to support an ecologic driving style were derived from literature. For each system a maximum 100-word description and an image that illustrated the system (see chapter 5 for details) were made. The descriptions were formulated neutrally and the illustrations used the same graphical style. To improve comprehensibility both text and graphics were presented to 3 fellow researchers and based on their suggestions reformulated and redesigned.

For the assessment of the persuasive interface user acceptance a questionnaire was developed that included the following items: Based on the TAM by Davis and Venkatesh two questions asked for Behavioural Intention of Use (BI). The scales for Perceived Usefulness (U) and Perceived Ease of Use (EOU) consisted of four questions each (e.g. Assuming I had access to ..., I intend to use it.). The TAM questionnaire proved to be very suitable for assessing the user acceptance, but does not provide detailed information on the reasons why a system is rated high or low. Therefore three additional questions were added that were computed separately from the TAM analysis and were expected to give further insights on the perception of the systems. One question asked for an expected disturbance (Disturbance) by the system. The second additional question addressed perceived security risks (Risk) caused by a system usage while driving. Question three was pointed towards the suitability of the system to serve its purpose, namely to support drivers in a more ecological driving behaviour (Suitability).

The five presented systems were randomized in their order of presentation to avoid biases. Additionally, the questions in the questionnaire were counterbalanced for every system to avoid artefacts caused by the question order. While the same questions were asked for each of the five systems, participants also filled out questions before they were confronted with the systems. These questions asked for gender, age, car usage frequency (driving) and the duration of the driving license ownership. Nine questions were asked for the general attitude towards new technology (Attitude). Two final questions were asked for a ranking of the systems from 1-5 and how easy it was to imagine the use of the described systems. The questionnaire was distributed in the form of an online questionnaire and communicated over various mailing lists in order to reach an audience as broad as possible.

\section{PERSUASIVE INTERFACES}

To evaluate the acceptance of future in-car interaction approaches for the purpose of persuading car drivers to drive in a more economic way, we decided to research already existing - but not yet deployed - approaches. This gave us the possibility to focus on studying UA rather than the design of new solutions. We therefore have accomplished an extended literature research. We aimed at identifying different approaches from academic as well as commercial sources, which are designed to support more fuelefficient driving. The systems were chosen based on two preconditions. First, the systems had to be in a pre-prototype stage. Second, the systems had to be understandable and imaginable by users who took part in the study.

After identifying several approaches from various sources, we decided to extract five different designs all fostering fuel-efficient driving. Each system presented in the following sections is based on already existing ideas but was redesigned by the authors, resulting in the fact that each system combines different properties of earlier identified approaches. The five systems were described in an online survey including the visualizations presented in this paper. Although most of the identified systems from the literature are illustrated with high-fidelity graphics, we decided to create our own graphical representations of the system to be more consistent in the representation of the different approaches. The following subsections characterise these five approaches. For each system, we first provide a description including a figure. Both the text and the figure resembles the information, which was given in the online questionnaire. Secondly, we describe systems from industry, which motivated our approaches. Thirdly, we discuss persuasive aspects of each system.

\subsection{Automatic Eco System (EcoMatic)}

\section{Description}

The Automatic Eco System (EcoMatic) is a fully automated in-car appliance supporting fuel-efficient driving. The system can be manually activated and deactivated by pressing an EcoButton (see Figure 1). When the system is operating, it automatically reduces fuel consumption by adjusting various parameters within the car. As an example, the automatic climate control is switched into an economy mode and the engine is automatically changed into an idle mode at red traffic lights. At the end of each trip the amount of saved fuel in comparison to the standard fuel consumption is shown (see Figure 1).

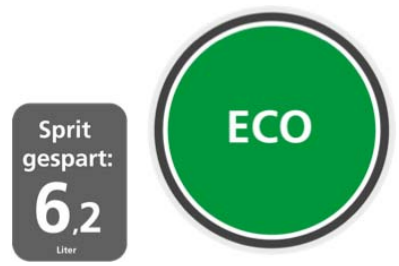

Figure 1: EcoMatic. Eco Button (right) and feedback display (left). English translation: "Fuel saved: 6.2 litres"

Motivation

This system is motivated by Honda's ECON Mode button, which is part of Honda's Ecological Drive Assist System [11]. The ECON Mode automatically achieves energy-saving control of the air-conditioning unit and extends the idle stop time. Contrary to our system, it does not provide any information about the amount of saved fuel during the last trip. Toyota plans to equip their new hybrid Prius with three driving modes: ECO (low acceleration power), EV (medium acceleration power, electric-only), and POWER (high acceleration power) [25]. These modes have influence on the acceleration pedal sensitivity.

\section{Discussion}

Since the automatic Eco System is operating autonomous, without any user input (except switching it on an off), it is not designed to change the operators driving behaviour. The persuasive element in this system is the displayed information at the end of each trip. This information aims at persuading the driver to use the EcoMatic by showing the concrete benefits of the system. The feedback relevant for economical driving is given once after a trip. One property with this system worth considering is that it might affect the user's comfort level, e.g. by switching the airconditioning in an economy mode. 


\subsection{Eco Accelerator Pedal (EcoPedal)}

\section{Description}

The Eco Accelerator Pedal (EcoPedal) is similar to a traditional acceleration pedal with one distinction: It aims at reducing fuel consumption by simply pushing back against the driver's foot when it detects wasteful acceleration (see Figure 2). This means that the driver feels an increased pressure against his foot, when for instance - he wants to push down the EcoPedal to its limit. Nevertheless, it is always possible for the driver to push down the pedal as far as he wants, even if the fuel consumption is wasteful.

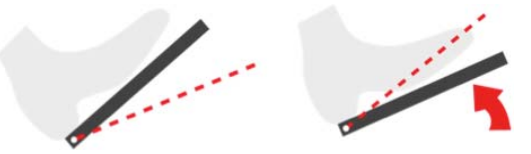

Figure 2: EcoPedal. Dotted red line: border of fuel economy deterioration. Left indicates an economical; right a wasteful acceleration with increased pedal pressure.

\section{Motivation}

A similar system has been introduced by Nissan [19]. Their ecopedal system activates a counter pushback control mechanism if the system detects excess pressure, each time the driver steps on the accelerator. The optimum acceleration rate is calculated using data on the rate of fuel consumption and transmission efficiency during acceleration and cruising.

\section{Discussion}

The EcoPedal aims at changing driver's behaviour by providing feedback at the right moment (when the driver is going to accelerate the car at cost of high fuel consumption) at the right place (within the car at the acceleration pedal itself). Since both the right moment and the right place are crucial elements of persuasive technologies [7], the EcoPedal seems to be a promising approach to assist drivers to become more fuel-efficient. On the other side, one has to keep in mind that the acceleration pedal is one of the most important interfaces for the driver's primary task.

\subsection{Eco Speedometer (EcoSpeedometer)}

\section{Description}

The EcoSpeedometer provides real-time fuel-efficient driving guidance. It is a display seamlessly integrated into the traditional speedometer providing visual feedback whether the driver is driving fuel-efficient at the moment or if the current driving style is wasteful. When driving fuel-efficient, the EcoSpeedometer glows green, when driving inefficiently, it glows orange (see Figure 3).
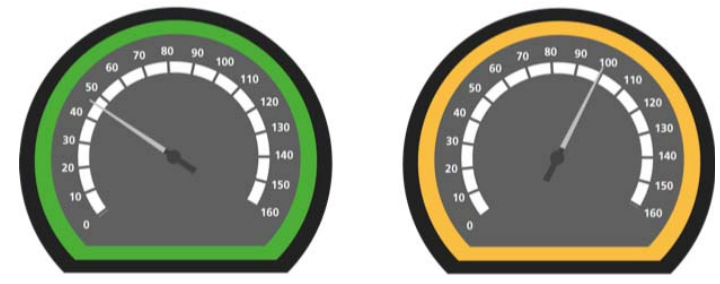

Figure 3: EcoSpeedometer. Left (green) indicates an economical; right (orange) a wasteful driving behaviour.

\section{Motivation}

Real-time visual feedback systems on the momentary driving behaviour have been proposed by several car manufacturers. Honda's Ambient Meter [11] is the background on the speedometer, which notifies the driver of the current driving conditions using colour (green for high fuel-efficient driving, blue-green for moderate fuel-efficiency and blue for wasteful driving). The Nissan eco-driving indicator [19] supports the above-described eco-pedal system. Incorporated on the instrument panel it glows green when the driver is driving within the optimal fuel consumption range. Toyota's Hybrid System Indicator [25] display also indicates whether the driving style is within an economical range.

\section{Discussion}

The EcoSpeedometer resembles the EcoPedal in the way that it aims at changing the driver's momentary driving behaviour by providing instant feedback. Contrary to the EcoPedal, the feedback of the EcoSpeedometer is persistent. It therefore provides positive feedback (green light). Additionally, we assume it to be not as distracting for the driver's main task as the EcoPedal. On the other hand, it might not be intuitively clear for the driver how to perform a more fuel-efficient driving style since the feedback is rather abstract.

\subsection{Eco Display (EcoDisplay)}

\section{Description}

The EcoDisplay visualizes the fuel-efficiency accumulative for the current trip by displaying a set of green leaves. The more fuelefficient the driving is, the more green leaves are shown (see Figure 4). When the driving habits become wasteful again, leaves begin to vanish. For each trip, an EcoScore is calculated and displayed at the end of each trip together with information on mileage and average fuel consumption. Additionally, a ranking of comparable trips is shown (see Figure 4). This EcoScore gives the driver the possibility to compare different trips or to compete against other drivers.
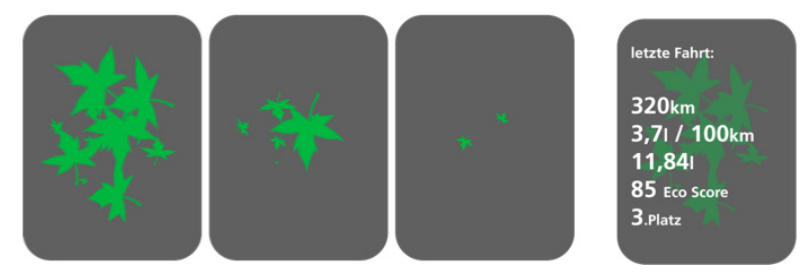

Figure 4: EcoDisplay. From left to right: high, medium, and low economic driving style, feedback display. English translation "Last trip: 320 km, 3.7 1/100 km, 11.84 litres, 85 Eco Score, $3^{\text {rd }}$ rank."

\section{Motivation}

A similar system was introduced by Ford with their SmartGauge with EcoGuide dashboard [8]. Long-term fuel efficiency is displayed by "growing leaves" and vines. The growing amount of leaves creates a visual reward for the driver's efforts to drive more fuel-efficient. When the car is turned off, summary information from the just-completed trip, as well as long-term comparative data, is displayed. Honda's Ecological Drive Assist System [11] includes the Eco Guide, which shows growing leaves in three stages as driving practices become more fuel-efficient over time. At the end of each trip, an Eco Score shows the drive cycle results, as well as "lifetime results", represented as leaves on the Eco Guide. The Multi-Information Display allows drivers to view fuel economy figures for the past three trips, as well as instantaneous and average fuel economy statistics.

\section{Discussion}

The EcoDisplay represents the most playful approach of the five presented systems. The visualization using leaves as a reward for 
fuel-efficient driving serves as a symbol for a greener earth. The possibility to reach high-scores and to compare the individual driving performance on an eco-scale with other drivers wants to turn driving into a green game. Contrary to the EcoSpeedometer, which also provides a visual feedback on driving behaviour, the EcoDisplay does not immediately react to wasteful driving behaviour but shows fuel efficiency over a certain period over time - in this case the duration of the last trip. It therefore aims at a long-term behaviour change by providing information over a longer driving cycle.

\subsection{Eco Advisor (EcoAdvisor)}

\section{Description}

The EcoAdvisor analysis driving behaviour along with car specific status information and presents hints to foster a more fuel-efficient driving. The hints are presented verbally before a trip or during driving in appropriate moments utilizing the in-car entertainment system (see Figure 5). Hints regarding the status information of the car are, for example, to increase the tire pressure to a certain amount or to remove unnecessary weight from the car. Hints to improve fuel-efficient driving are e.g. to switch into a higher gear or not to drive at full throttle.

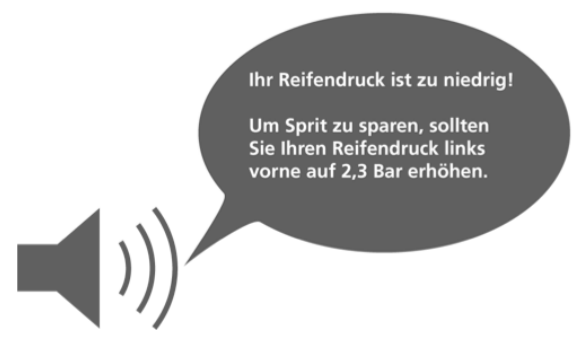

Figure 5: EcoAdvisor. English translation: "Your tyre pressure is too low! To save fuel you should increase the air pressure of your front left tyre to 2.3 bar."

\section{Motivation}

The idea of the EcoAdvisor was encouraged by Fiat's eco:Drive system [6], which is a computer application with the aim of improving fuel-efficient driving. While the car is being driven, data pertaining to the vehicle's fuel consumption, exhaust emissions, and the driver's acceleration, braking and shifting patterns are recorded on a flash drive. Later, the data is downloaded to a PC equipped with the eco:Drive software, which can suggest specific changes to driving behaviour [10].

\section{Discussion}

The EcoAdvisor is the only auditory system presented here. It gives feedback in form of concrete improvement suggestions at appropriate moments. From a persuasive perspective, concrete improvements and the right timing of giving hints are desirable. Using the auditory channel has the advantage of avoiding visual cluttering, but does not allow the user to actively choose the moment of information gathering.

\subsection{Persuasive System Attributes}

As stated above all five systems have in common that they are aiming at persuading driver's to change their driving behaviour to drive more fuel-efficient. Besides this, we chose interfaces with different persuasive attributes.

The most obvious difference lies in the sensory channel addressed by the systems. The EcoPedal gives a tactile feedback, whereas the EcoAdvisor an auditory one. The EcoDisplay, the
EcoSpeedometer and the EcoMatic, by means of the feedback display at the end of each trip, address the visual channel. Another difference can be found when looking at how the systems are integrated into the dashboard. Three systems make use of already existing in-car interfaces (EcoPedal: acceleration pedal, EcoSpeedometer: speedometer, EcoAdvisor: sound system), whereas the EcoMatic and the EcoDisplay create new interfaces.

One of the most important characteristics of persuasive strategies is the intervention at the opportune moment, which is also referred to as kairos [7]. A persuasive intervention at this moment increases the likelihood of a successful outcome, resulting in the desired behavioural change. Regarding kairos, three different kinds of systems can be found. Two systems (EcoPedal, EcoAdvisor) give feedback on the driving behaviour in appropriate moments (at the exact moment when the driver is going to drive wasteful), two systems (EcoSpeedometer, EcoAdvisor) give constant feedback, whereas the EcoMatic gives feedback only once after each trip.

Another distinction can be seen in the style of feedback and whether it aims at changing the momentary or long time driving behaviour. The EcoAdvisor and the EcoPedal provide concrete suggestion on how to drive more fuel-efficient, whereas the other three approaches (EcoMatic, EcoSpeedometer, EcoDisplay) offer only generic information. The EcoPedal, the EcoSpeedometer, and the EcoAdvisor give feedback on momentary driving behaviour, whereas the EcoMatic and the EcoDisplay provide feedback over a longer period of time.

\section{RESULTS}

57 participants ( 31 female, 26 male) took part in the study. They were recruited through email invitation and filled out the online survey. The questionnaire was created using LimeSurvey (www.limesurvey.org), it was online for 2 weeks and it took approximately 10 minutes to answer the questions. All of our participants owned a driver license (duration range: 1 to 40 years), the average age of the participants was 30.04 years $(\mathrm{SD}=9.51)$ with a range from 19 to 58 years. Half of our participants stated that they use a car at least several times a week (57.2\%), $21.4 \%$ use a car at least several times a month and $21.4 \%$ use a car less frequent.

At first, we computed the TAM scales Behavioural Intention of Use (BI), Perceived Usefulness (U) and Perceived Ease of Use (EOU) for each system and checked their reliability. To measure the internal consistency of the scales, we computed the Cronbach Alphas for each scale. They ranged for BI from .883 to .940 , for $\mathrm{U}$ from .880 to .939 and for EOU from .841 to .941 , therefore indicating a generally high internal consistency of the individual scales.

In terms of research goal 1 (RG1), we conducted a repeatedmeasure ANOVA with system as within-subject factor and the Behavioural Intention of Use as dependent variable in order to assess the significance of possible differences. The ANOVA showed a significant main effect for the within-subject factor $\left(\mathrm{F}(3.30,152.203)=20.061, \mathrm{p}<.001, \eta^{2}=.304\right)$. To show between which systems differences emerge, a post-hoc-test (Bonferonni) was conducted. It indicated that the EcoPedal was rated significantly $(\mathrm{p}<.01)$ lower than the EcoMatic, EcoSpeedometer and the EcoDisplay, but not lower than the EcoAdvisor. The EcoSpeedometer was significantly $(\mathrm{p}<.01)$ higher rated than the EcoDisplay, EcoAdvisor and EcoPedal. The EcoMatic did not 
differ from the EcoSpeedometer but was rated significantly $(\mathrm{p}<.01)$ higher than the other systems.

Similar findings emerged for the scale Perceived Usefulness i.e. there was a significant difference between the systems $(\mathrm{F}(4,180)=$ $\left.12.206, \mathrm{p}<.01, \eta^{2}=.213\right)$. Firstly, the EcoPedal was rated lowest again in comparison to all the other systems and the difference was significant $(\mathrm{p}<.01)$. Secondly, the EcoMatic and the EcoSpeedometer were perceived significantly more useful than the other systems $(\mathrm{p}<.01)$.

At last, we compared the Perceived Ease of Use score across the different systems and found again a significant main effect $\left(\mathrm{F}(4,176)=15.427, \mathrm{p}<.01, \eta^{2}=.260\right)$. In this case, the EcoSpeedometer was perceived as the easiest system to use $(\mathrm{p}<.01)$, whereas no difference between the other systems emerged. For an illustration of the findings see Figure 6.

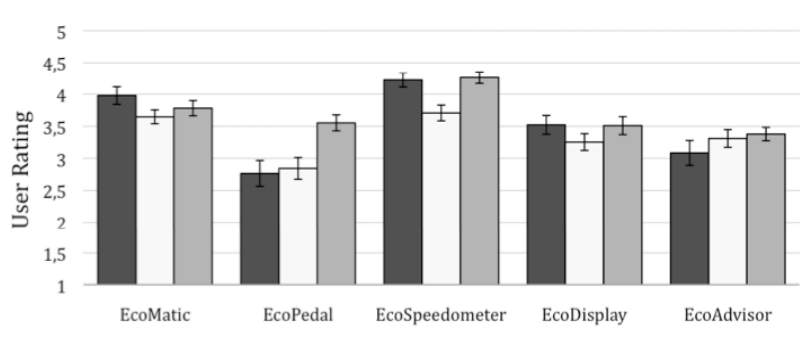

- Behavioral Intention to Use $\square$ Perceived Usefulness $\square$ Perceived Ease of Use

Figure 6: Mean Scores of Behavioural Intention of Use (BI), Perceived Usefulness (U) and Perceived Ease of Use (EOU) for the different systems (including error bars)

Analyzing which individual factors influenced the participants' evaluations, we concentrated on the best (EcoSpeedometer) and worst (EcoPedal) rated systems. Not surprisingly the rating of all three factors (Risk, Disturbance and Suitability) were highly similar to the TAM rating, which seems to be a cue for face validity. The EcoSpeedometer was considered both as least fraught of risk, least disturbing, and most suitable for assisting economic driving. The EcoPedal was rated as relative high in risk and disturbance. The Suitability factor was rated above average but nevertheless significantly lower than the EcoSpeedometer (see also Figure 7).

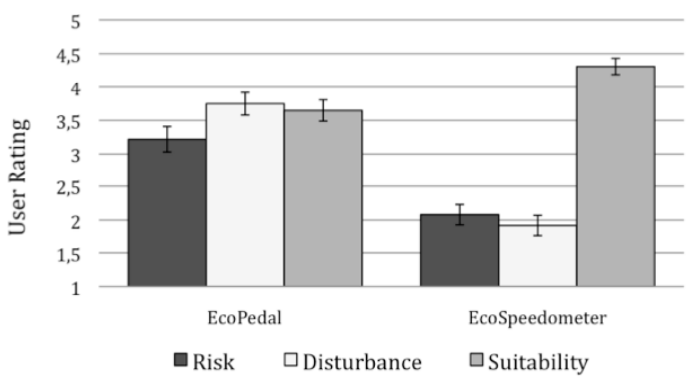

Figure 7: Mean Scores of Risk, Disturbance and Suitability while system usage for EcoPedal and EcoSpeedometer

For both systems we conducted linear regressions to predict the individual TAM scores. As possible predictors, we included age, gender, frequency of driving a car, the scale concerning general attitude toward car technology and questions toward the possible disturbance (Disturbance), risk for safety (Risk) and perceived suitability of the system (Suitability). All of these predictors were entered in the regression equation using the stepwise method.

For the prediction of the three TAM scales of the EcoPedal, Disturbance and Suitability emerged as best and only predictors explaining $68.4 \%$ of the variance. While Disturbance had a strong negative influence $(\beta=-.576)$, the Suitability had a moderate positive effect $(\beta=.319)$ meaning that the indicated Intention to Use (BI) was higher when the user did not perceive the system as disturbing and the user expected the system to be suitable supporting economic driving. A very similar pattern emerged for the Perceived Usefulness scale (Suitability: $\beta=.490$; Disturbance: $\beta=-.444$ ) explaining $73.8 \%$ of the variance. However, there was no significant effect of Disturbance on Perceived Ease of Use, but again an effect of Suitability emerged $(\beta=.581)$.

For the EcoSpeedometer, the predictors Suitability $(\beta=.762)$ and general attitude towards new technology (Attitude) $(\beta=.256)$ emerged as the best predictors for BI. For U, we found Suitability $(\beta=.644)$ and Attitude $(\beta=.278)$ to be predictive. EOU was predicted solely through Attitude $(\beta=.330)$. The effects of the other postulated predictors did not reach significance and were generally low $(|\beta|<.17)$. The results of the linear regression are also summed up in more detail in Table 1.

Table 1. Predictors for the factors Behavioural Intention to use (BI), Perceived Usefulness (U) and Perceived Ease of Use (EOU) of the EcoPedal $(* \mathbf{p}<.05 ; * * \mathbf{p}<.01)$

\begin{tabular}{|l|c|c|c|}
\hline \multicolumn{4}{|c|}{ EcoPedal } \\
\hline \multicolumn{1}{|c|}{ Factor } & Predictor & $\boldsymbol{\beta}$ & t \\
\hline \multirow{2}{*}{$\mathrm{BI}\left(\mathrm{R}^{2}=.684\right)$} & Disturbance & -.576 & $-4.888^{* *}$ \\
\cline { 2 - 4 } & Suitability & .319 & $2.712^{* *}$ \\
\hline \multirow{2}{*}{$\left(\mathrm{R}^{2}=.738\right)$} & Suitability & .490 & $4.568^{* *}$ \\
\cline { 2 - 4 } & Disturbance & -.444 & $-4.143^{* *}$ \\
\hline EOU $\left(\mathrm{R}^{2}=.338\right)$ & Suitability & .581 & $4.845^{* *}$ \\
\hline \multicolumn{4}{|c|}{ EcoSpeedometer } \\
\hline \multirow{2}{*}{$\mathrm{BI}\left(\mathrm{R}^{2}=.672\right)$} & Suitability & .762 & $9.082^{* *}$ \\
\cline { 2 - 4 } & Attitude & .256 & $3.055^{* *}$ \\
\hline \multirow{2}{*}{$\mathrm{U}\left(\mathrm{R}^{2}=.507\right)$} & Suitability & .644 & $6.255^{* *}$ \\
\hline & Attitude & .278 & $2.700^{* *}$ \\
\hline EOU $\left(\mathrm{R}^{2}=.09\right)$ & Attitude & .330 & $2.373^{*}$ \\
\hline
\end{tabular}

Concerning our third research goal (RG3), whether an online survey is an apt methodology for evaluating persuasive in-car technologies, we would argue that our approach seems to be promising (see Fehler! Verweisquelle konnte nicht gefunden werden.).

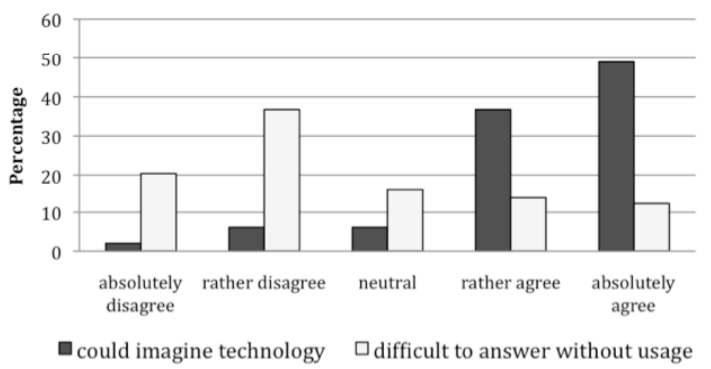

Figure 8: Frequency distribution of answers to the questions "I could imagine the presented technologies because of the description and pictures" and "It was difficult to answer the questions without actually using the technology" 
The major part of the participants $(85.7 \%)$ indicated that they could imagine the technology after reading the description and seeing the pictures. The opinion toward the question whether it was difficult to answer the questions without actual usage of the technology was more heterogeneous: although $57.1 \%$ disagreed with this statement, $22.8 \%$ answered affirmative to this question.

As mentioned in chapter 4 we asked participants at the end of the survey to rank the systems from 1 to 5 (see Figure 9). The figure shows how often each system was assigned to rank 1-5. Comparing the results of the TAM questionnaire and the participants' ranking of the different systems, it can be concluded that parallels between these two measures emerge: the EcoSpeedometer was evaluated best (median rank of 1 and mean rank of 1.75) and the EcoPedal worst (median rank of 4 and mean rank of 3.977) in the direct evaluation, as well as in the TAM. It has to be noted that the EcoAdvisor's mean and median rating did not differ much from the EcoPedal, which is consistent with the findings from the TAM score that the differences of Behavioural Intention to Use and Perceived Ease of Use between these two systems were not significant.

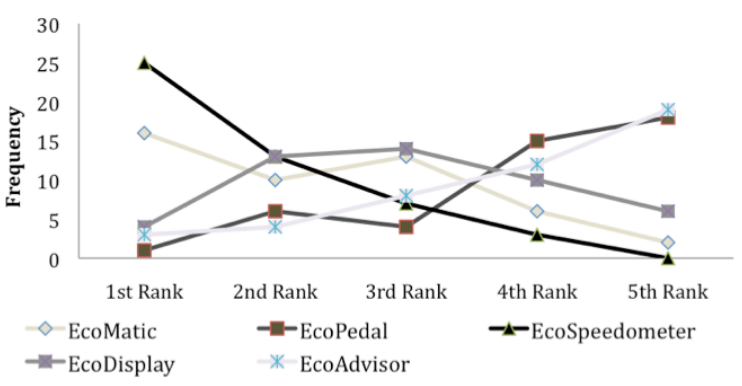

Figure 9: Ranking frequency of the systems.

\section{DISCUSSION}

In this section we will discuss the results of our study for each research goal:

\section{Research Goal 1 (RG1)}

RG1 was to evaluate the user acceptance of persuasive in-car interfaces that are designed to support a fuel-efficient driving style.

For all five systems differences regarding User Acceptance (UA) as well as the three UA factors Behavioural Intention to Use (BI), Perceived Usefulness (U), and Perceived Ease of Use (EOU) were found. All factors were rated positive for the EcoMatic, the EcoSpeedometer, the EcoDisplay as well as the EcoAdvisor. Only for the EcoPedal Behavioural Intention to Use (BI) and Perceived Usefulness (U) were rated negatively.

The user acceptance for the EcoSpeedometer was rated highest for all three factors, followed by the EcoMatic. Especially the intention to use (BI) and the perceived usefulness for these two systems were rated significantly higher than for the other systems. The EcoDisplay as well as the EcoAdvisor were rated almost the same on the scales $U$ and EOU, but the EcoDisplay was rated higher than the Ecoadvisor on the intention to use scale. The EcoPedal was rated lowest on the factors intention to use (BI) and perceived usefulness (U). Compared to these scales, the perceived ease of use (EOU) factor is rated positive and rather high. This leads to the assumption that the users would find it comprehensible and easy to use, but neglect its usefulness and would rather not use it. This finding goes along with Davis and Venkatesh's [3] assumption, that the factor U strongly affects BI but has only a low effect on EOU.

Due to the fact that most systems did not require sophisticated interactions, we expected that the factor perceived ease of use (EOU) would be rated higher for all systems than the other factors. This was not the case in our study. We assume that the users did not rate only the handling of the system but also the transparency of system behaviour. This assumption is strengthened by the fact that the EOU for the EcoSpeedometer, a bicolour interface with a plain behaviour, was rated relatively high.

Research Goal 2 (RG2)

RG2 was to analyse how driver's general attitude toward technology in cars, sociodemographic variables and factors like driver properties and expectations toward the technology concerning safety, disturbance and assistance have influence on the user acceptance.

Sociodemographic variables generally played no role for the systems' evaluations. This could be partly due to the fact that the influence of the questions concerning disturbance, safety and expectancy of assistance were explaining a major part of the variance. Indeed, we found a high influence of expected disturbance and expectancy of assistance. Participants felt especially disturbed by systems with tactile and/or auditory feedback, raising the question whether this finding can be generalized and should be considered in future research and design. Safety issues were mostly no problem for our participants, but nevertheless it has to be noted that a rating of 3 (see Figure 7) or more seems problematic for a system. It remains unclear whether the systems were indeed rated as a possible risk while driving a car, or this finding can be explained as a result of a more general halo effect [24]. This would mean that the appearance and evaluation of an object in other dimensions has an effect on the rating of other system's properties.

\section{Research Goal 3 (RG3)}

RG3 was to research if the usage of an online TAM questionnaire for in-car persuasive interfaces in a pre-prototype level would lead to results that support future design decisions.

The fact that we gained different, interpretable results for the different systems encourage our assumption that user acceptance in a pre-prototype phase of persuasive in-car interfaces using the TAM with different scenarios in an online survey is reasonable. $85.7 \%$ of the users indicated that they could imagine the technology after reading the description and seeing the pictures. $26.5 \%$ found it challenging to answer questions about the systems without actually using them. It seems to be an economic approach with ecological validity to gain early feedback, which seems especially important for prototypes in development. However, this approach does not change the importance of other methods (e.g. focus-groups) to gain valuable insights on users' thoughts about technology of tomorrow.

\section{FUTURE WORK}

To support the reliability of our results firstly focus groups and secondly the evaluation of hands-on prototypes will be conducted. A comparison of our results and the real driving experience using the systems would be interesting. Furthermore it seems promising to investigate the relatively more negative rating of the EcoPedal 
and EcoAdvisor. The negative ratings could be partly due to the fact that feedback was given tactile and auditory. Whereas the first feedback may be interpreted by users as working against one's own intention, the latter may be negative because it can be interpreted as similar to the nagging and complaining of a codriver. Our next steps will be to classify the systems based on system properties like feedback style. Another approach will be to systematically covariate the systems persuasive properties in order to identify which persuasive strategy is most accepted by the users. Additionally to that, a closer look will be taken on the drivers' general attitude towards technology and potential influences on user acceptance.

\section{REFERENCES}

[1] Ablassmeier, M., Poitschke, T., \& Rigoll, G. 2006. A new approach of a context-adaptive search agent for automotive environments. In Proc. CHI '06 Extended Abstracts, New York, NY, USA, 1613-1618. ACM.

[2] Comte, S. Wardman, M., Whelan, G. 2000, Drivers acceptance of automatic speed limiters: implications for policy and implementation, Transport Policy 7(4), 259-267

[3] Davis, F. D. \& Venkatesh, V. 2004. Toward preprototype user acceptance testing of new information systems: implications for software project management. Engineering Management, IEEE Transactions on, 51(1), 31-46.

[4] Davis, F. D. 1989. Perceived Usefulness, Perceived Ease of Use, and User Acceptance of Information Technology. MIS Quarterly, 13(3), 318+.

[5] Dillon, A. \& Morris, M. G. 1996. User acceptance of new information technology: theories and models. http://dlist.sir.arizona.edu/1261/

[6] Fiat, eco:Drive, http://www.fiatecodrive.com (last accessed on $25^{\text {th }}$ May 2009)

[7] Fogg, B.J. 2003. Persuasive Technology: Using Computers to Change What We Think and Do. Morgan Kaufman, San Francisco, CA, USA.

[8] Ford, SmartGauge with EcoGuide, http://media.ford.com/images/10031/SmartGauge.pdf (last accessed on $25^{\text {th }}$ May 2009)

[9] Graf, S., Spiessl, W., Schmidt, A., Winter, A., and Rigoll, G. 2008. In-car interaction using search-based user interfaces. In Proc. CHI '08. ACM, New York, NY, 1685-1688.

[10] Green Car Advisor, http://blogs.edmunds.com/greencaradvisor/2009/04/ecodriving-systems-now-your-car-can-gently-nag-you-intobeing-more-fuel-wise.html (last accessed on $25^{\text {th }}$ May 2009)

[11] Honda, Ecological Drive Assist System, http://world.honda.com/news/2008/4081120EcologicalDrive-Assist-System/ (last accessed on $25^{\text {th }}$ May 2009)

[12] Hu, J., Winterboer, A., Nass, C. I., Moore, J. D., \& Illowsky, R. 2007. Context \& usability testing: user-modelled information presentation in easy and difficult driving conditions. In Proc. CHI '07, New York, NY, USA, 2007 1343-1346. ACM.
[13] Kantowitz, B. H., Hankowski, R. J., and Kantowitz, S.C. 1997. Driver acceptance of unreliable traffic information in familiar and unfamiliar settings. Human Factors 39, 164-176.

[14] Kern, D., Müller, M., Schneegaß, S., Wolejko-Wolejszo, L., $\&$ Schmidt, A. 2008. CARS - Configurable Automotive Research Simulator. In Automotive User Interfaces and Interactive Applications - AUIIA 08, Workshop at Mensch und Computer 2008.

[15] Kern, D., Schmidt, A., Arnsmann, J., Appelmann, T., Pararasasegaran, N., and Piepiera, B. 2009. Writing to your car: handwritten text input while driving. In Proc. CHI '09 Extended Abstracts. ACM, New York, NY, 4705-4710.

[16] Kim, S., Dey, A. K. 2009. Simulated augmented reality windshield display as a cognitive mapping aid for elder driver navigation. In Proc. CHI '09. ACM, New York, NY.

[17] Krum, D. M., Faenger, J., Lathrop, B., Sison, J., and Lien, A. 2008. All roads lead to CHI: interaction in the automobile. In Proc. CHI '08 Extended Abstracts. New York, NY. ACM.

[18] Kumar, M. and Kim, T. 2005. Dynamic speedometer: dashboard redesign to discourage drivers from speeding. In Proc. CHI '05 Extended Abstracts. New York, NY. ACM.

[19] Nissan, ECO Pedal, http://www.nissanglobal.com/EN/NEWS/2008/ STORY/080804-02-e.html (last accessed on $25^{\text {th }}$ May 2009)

[20] Pace, T., Ramalingam, S., and Roedl, D. 2007. Celerometer and idling reminder: persuasive technology for school bus eco-driving. In Proc. CHI '07 Extended Abstracts. New York, NY, 2085-2090. ACM.

[21] Schmidt, A., Boll, S., Spieß1, W., \& Kranz, M. 2008. Automotive User Interfaces and Interactive Applications. In Workshop at the Conference Mensch und Computer 2008.

[22] Siewiorek, D., Smailagic, A., \& Hornyak, M. 2002. Multimodal Contextual Car-Driver Interface. Multimodal Interfaces, In Proc. Multimodal Interfaces, 367-373. IEEE.

[23] Tester, J., Fogg, B., and Maile, M. 2000. CommuterNews: a prototype of persuasive in-car entertainment. In Proc. CHI'00 Extended Abstracts. New York, NY, 24-25. ACM.

[24] Thorndike, E. L. 1920. A constant error in psychological rating. Journal of Applied Psychology, 4, 25-29.

[25] Toyota, $3^{\text {rd }}$ Generation Prius, http://www.toyota.com/ vehicles/minisite/newprius/ (last accessed on $25^{\text {th }}$ May 2009)

[26] Várhelyi, A., Hjälmdahl, M., Risser, R., Draskóczy, M., Hydén, C., \& Almqvist, S. 2002. The effects of Large Scale use of active accelerator pedal in urban areas. In International Cooperation of Theories and Concepts in Traffic safety (ICTCT) Workshop, Nagoya, Japan , 2002.

[27] Venkatesh, V., Morris, M.G., Davis, G.B., Davis, F.D. 2003 "User acceptance of information technology: toward a unified view," MIS Quarterly (27:3). 425-478.

[28] Wochinger, K., Boehm-Davis, D. 1997. Navigational preference and driver acceptance of advanced traveler information systems. In Ergonomics and Safety of intelligent Driver interfaces. NJ, 345-362. 